\title{
Benchmark
}

\section{Notes on Transient Host Range Selection for Engineering Vaccinia Virus Strain MVA}

BioTechniques 33:186-188(July 2002)

\author{
David C. Tscharke and \\ Geoffrey L. Smith \\ Imperial College of Science, \\ Technology, and Medicine, \\ London, UK
}

Modified vaccinia virus Ankara (MVA) is a highly attenuated strain of vaccinia virus (VV) that is a candidate vector for recombinant vaccines and also a useful tool for gene expression in cell culture (10). MVA replicates in some avian cells, such as primary chick embryo fibroblasts (CEFs), and also the hamster kidney fibroblastoid line, BHK$21(3,5)$. This makes MVA safe but also limits the methods for selection of recombinant viruses. Original vectors for making recombinant MVAs (rMVAs) used $\beta$-galactosidase or $\beta$-glucuronidase and required visual selection of recom binants $(2,10)$. However, the VV K1L gene can expand the host range of MVA to include $\mathrm{RK}_{13}$ cells, a rabbit kidney fibroblast line (11), and this was exploited in a transient host range selection scheme for making rMVAs (9). We have developed a similar scheme using a sim pler plasmid vector that reduces the number of cloning steps required to engineer defined loci of the MVA genome and leaves no foreign sequences in the final virus. This plasmid contains $\mathrm{K} 1 \mathrm{~L}$ outside, instead of between the MVA targeting sequences. Intermediate viruses, which are derived from single crossover events and contain the entire plasmid, are selected on $\mathrm{RK}_{13}$ cells and subsequently are resolved (by intra-genomic recombination) either to the desired recombinant or wild-type MVA af- ter growth on CEF cells. This approach has been used with other selectable markers to engineer other VV strains (6). We used this scheme to make several rMVAs each deleted in a different gene and containing no non-MVA sequences (not shown). A lack of nonMVA sequences is desirable in viruses for use in humans.

A potential problem with the use of $\mathrm{K} 1 \mathrm{~L}$ as a selectable marker is that MVA contains approximately one-third of the K1L gene (1), which in theory allows targeting of a K1L-containing plasmid to the $\mathrm{K} 1 \mathrm{~L}$ region rather than elsewhere. K1L in MVA is defective in two places; part of the core sequence of the promoter (4) is deleted, and the latter two-thirds of the coding sequence have been removed by a large deletion (Figure 1A). These defects at both ends of the gene mean that recombination events between the K1L coding sequence of a plasmid and the intact third of the gene in MVA should not yield a functional K1L gene. However, if sequences upstream of the K1L promoter are included, then a single recombination between these sequences in the plasmid and the MVA genome can produce a rMVA with a functional K1L gene. We considered integrations into short sequences to be rare events, and our original plasmid vector, $\mathrm{pK} 1 \mathrm{~L}$, was made by replacing the 553-bp SspI fragment of pBluescript ${ }^{\circledR}$ (Stratagene, La Jolla, CA, USA) (KS) with the K1L coding sequence from VV strain WR, including its natural promoter and approximately $60 \mathrm{bp}$ sequence upstream (similar to Reference 9). However, by diagnostic PCR, we found that in some experiments more than half of our intermediate rMVAs made using $\mathrm{pK} 1 \mathrm{~L}$ based plasmids had insertions in the $\mathrm{K} 1 \mathrm{~L}$ region instead of, or as well as, into the targeted gene (not shown). An intermediate virus having two copies of the plasmid, one in the $\mathrm{K} 1 \mathrm{~L}$ region and one in the targeted region, can still resolve to the desired rMVA, but intermediates with the plasmid inserted only in the sequences upstream of the K1L promoter will always resolve to wildtype, increasing the background when screening for final recombinants.

For this reason, we redesigned our vector such that it contained no sequences upstream of the promoter (Figure 1A). These K1L sequences were amplified from VV WR DNA using primers: 5'-TATAAAAAATGAAAAAATATACACTAATTAG-3' and 5'-AATGAGTA AGACAATAGGAAATATATC-3'. As in $\mathrm{pK} 1 \mathrm{~L}$, this fragment was cloned between the SspI sites of pBluescript (KS). Two versions of the plasmid were isolated, pDRT1 and pDRT2, in which K1L is transcribed away from and towards the Bluescript multiple cloning site (MCS), respectively. The full MCS, and therefore the ability to use blue/white color selection ( $\beta$-galactosidase $\alpha$-complementation in E. coli), is retained in these vectors, facilitating the addition of different MVA targeting sequences with deletions or foreign genes inserted.

We tested pK1L and pDRT1, with and without added MVA targeting sequences, for their ability to integrate into the MVA genome and thus rescue the virus for growth on $\mathrm{RK}_{13}$ cells (Figure 1B). A cassette designed to delete the majority of the MVA184R (VV Copenhagen gene B16R) coding sequence from MVA was cloned into the MCS of pDRT1 and pK1L to make pDRT1d184 and pK1Ld184. This cas- 
sette comprised sequences flanking 184R (each >500 bp) and joined to create a large deletion covering $75 \%$ of the 184R open reading frame (nucleotides
162046-162775 of the MVA genome). In addition, pd184, which has MVA 184R targeting sequences but no K1L gene, was made, and, lastly, we used

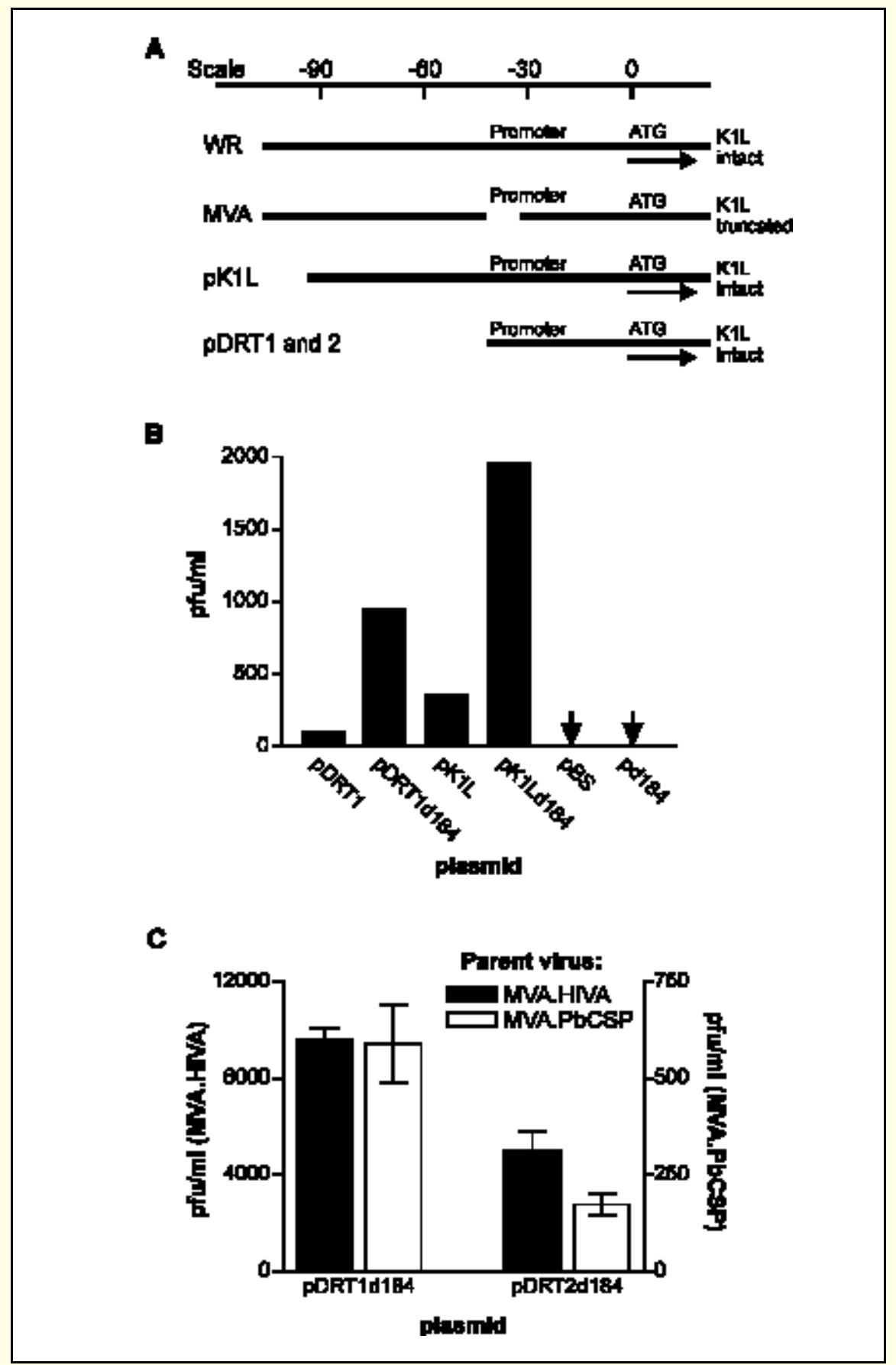

Figure 1. Use of plasmids containing K1L to generate rMVAs. (A) Comparison of K1L promoter region in VV WR and MVA and the extent of the WR sequence included in the plasmids pK1L and pDRT1/pDRT2. (B) The titer of MVA.HIVA able to grow on $\mathrm{RK}_{13}$ cells after transfection of infected CEF cells with the plasmids shown on the x-axis. Arrows, less than $1 \mathrm{pfu} / \mathrm{mL}$. (C) Effect of K1L orientation on ease of generation of recombinant MVAs. CEFs were infected with MVA.HIVA or MVA.PbCSP and then transfected with pDRT1d184 or pDRT2d184 (see x-axis). The average titers $(n=3, \pm \mathrm{SD})$ of recombinant progeny able to grow on $\mathrm{RK}_{4}{ }_{3}$ cells are plotted using left and right axis for recombinants of MVA.HIVA and MVA.PbCSP, respectively. 
pBluescript as a negative control. CEFs were grown to confluence overnight in a six-well plate and were infected with MVA.HIVA (7) at 0.05 focus forming units (ffu) (3) per cell for $2 \mathrm{~h}$ before being transfected with $1 \mu \mathrm{g}$ plasmid DNA using Lipofectin ${ }^{\circledR}$ according to the manufacturer's instructions (Invitrogen, Carlsbad, CA, USA). Two days after transfection, cells were scraped from the wells into the culture medium, and virus was released by three cycles of freezing and thawing and a sonication. The titer of recombinant progeny in each suspension was determined by plating serial 5-fold dilutions of the virus suspension on $\mathrm{RK}_{13}$ cells $(1 \mathrm{~mL}$ virus/well in six-well plates) overnight. After infection, the inocula were removed from the wells and replaced with medium containing 1\% low melting point agarose and $10 \%$ FBS. MVA. HIVA expresses $\beta$-galactosidase, allow ing visualization of plaques after three days of growth by the addition of a second layer of agarose-medium containing $0.4 \mathrm{mg} / \mathrm{mL} \mathrm{X}$-gal. After $6 \mathrm{~h}$, blue plaques were counted. As expected, no plaques were found on $\mathrm{RK}_{13}$ cells when pBluescript or pd184 were transfected into MVA.HIVA-infected cells. Surprisingly, all the K1L-containing plasmids generated rMVAs capable of growth on $\mathrm{R} \mathrm{K}_{13}$ cells, including pDRT1 that contains no other MVA sequences. While pDRT1 produced the smallest amount of recombinant progeny, some 9-fold less than the same plasmid with MVA184R sequences inserted, this result indicates that spurious integration into the K1L locus can still occur. In comparison, pK1L produced only 5fold less plaques on $\mathrm{RK}_{13}$ cells than pK1Ld184, indicating that pDRT1based plasmids are likely to give rise to less unwanted recombinants. We did not determine the structure of the rMVAs generated using pDRT1, but they might be formed by recombination between virus genomes that already contain copies of pDRT1. Alternatively, the first third of the K1L gene when coupled to a functional promoter may be able to rescue MVA for growth on $\mathrm{RK}_{13}$ cells. Whichever is the case, the only way to eliminate this problem is to use a parental MVA virus from which all K1L sequences have been removed.

Having made two vectors, pDRT1 and pDRT2, with the selectable marker in opposite directions, we were also able to test the effect of K1L orientation on ease of generating recombinant viruses (Figure 1C). After infecting six wells of CEFs with MVA.HIVA as above, $1 \mu \mathrm{g}$ pDRT1d184 and pDRT2d184 were each used to transfect three wells. Virus in each well was harvested separately, and recombinants were counted after growth on $\mathrm{RK}_{13}$ cells and X-gal staining (as described above). This experiment was repeated using a second rMVA, MVA.PbCSP (8) as the parent. Irrespective of parental virus, pDRT1d184 produced significantly more recombinant progeny than pDRT2d184 (Figure 1C). Possible explanations for this result include differences in efficiencies of (i) transfection into MVA-infected CEFs, (ii) recombination into the MVA genome, or (iii) growth of recombinants in CEFs. Thus, the orientation of K1L affects the efficiency with which recombinants can be generated. To our knowledge, this is the first observation of such an effect with any marker in the generation of recombinant VVs.

In conclusion, we present here an alternative transient host range selection scheme for making rMVAs by using the K1L gene of VV WR. This system has been used to demonstrate that the orientation of a selectable marker can influence the amount of recom binant progeny that can be obtained. Finally, we show that residual K1L sequences in MVA increase the background of unwanted recombination events when using K1L-based vectors, making screening of this region in resulting rMVAs important and suggesting the utility of a parental MVA with these sequences removed.

\section{ACKNOWLEDGMENTS}

This work has been supported by program grant no. 037575 from The Wellcome Trust. D.C.T. was supported by a Wellcome Trust Travelling Fellowship, and G.L.S. is a Wellcome Trust Principal Research Fellow.

\section{REFERENCES}

1.Antoine, G., F. Scheiflinger, F. Dorner, and F.G. Falkner. 1998. The complete genomic sequence of the modified vaccinia Ankara strain: comparison with other orthopoxviruses. Virology 244:365-396.

2.Carroll, M.W. and B. Moss. 1995. E. coli $\beta$ glucuronidase (GUS) as a marker for recombinant vaccinia viruses. BioTechniques 19:352356.

3.Carroll, M.W. and B. Moss. 1997. Host range and cytopathogenicity of the highly attenuated MVA strain of vaccinia virus: propagation and generation of recombinant viruses in a nonhuman mammalian cell line. Virology 238:198-211.

4.Davison, A.J. and B. Moss. 1989. Structure of vaccinia virus early promoters. J. Mol. Biol. 210:749-769.

5.Drexler, I., K. Heller, B. Wahren, V. Erfle, and G. Sutter. 1998. Highly attenuated modified vaccinia virus Ankara replicates in baby hamster kidney cells, a potential host for virus propagation, but not in various human transformed and primary cells. J. Gen. Virol. 79:347-352.

6.Falkner, F.G. and B. Moss. 1990. Transient dominant selection of recombinant vaccinia viruses. J. Virol. 64:3108-3111.

7.Hanke, T. and A.J. McMichael. 2000. Design and construction of an experimental HIV1 vaccine for a year-2000 clinical trial in Kenya. Nat. Med. 6:951-955.

8.Schneider, J., S.C. Gilbert, T.J. Blanchard, T. Hanke, K.J. Robson, C.M. Hannan, M. Becker, R. Sinden, et al. 1998. Enhanced im munogenicity for CD8+ T cell induction and complete protective efficacy of malaria DNA vaccination by boosting with modified vaccinia virus Ankara. Nat. Med. 4:397-402.

9.Staib, C., I. Drexler, M. Ohlmann, S. Wintersperger, V. Erfle, and G. Sutter. 2000. Transient host range selection for genetic engineering of modified vaccinia virus Ankara. BioTechniques 28:1137-1148.

10.Sutter, G. and B. Moss. 1992. Nonreplicating vaccinia vector efficiently expresses foreign genes. Proc. Natl. Acad. Sci. USA 89:1084710851.

11.Sutter, G., A. Ramsey-Ewing, R. Rosales, and B. Moss. 1994. Stable expression of the vaccinia virus $\mathrm{K} 1 \mathrm{~L}$ gene in rabbit cells com plements the host range defect of a vaccinia virus mutant. J. Virol. 68:4109-4116.

Received 22 February 2002; accepted 1 April 2002.

Address correspondence to:

Professor Geoffrey L. Smith

Room 333, Department of Virology

The Wright-Fleming Institute

Faculty of Medicine

Imperial College of Science, Technology and Medicine

St. Mary's Campus

Norfolk Place, London, W2 IPG, UK

e-mail:glsmith@ic.ac.uk

For reprints of this or any other article, contact Reprints@BioTechniques.com 